\title{
DW-MRI-Guided Dose Escalation Improves Local Control of Locally Advanced Nasopharyngeal Carcinoma Treated with Chemoradiotherapy
}

This article was published in the following Dove Press journal: Cancer Management and Research

\author{
Yecai Huang $\mathbb{D}^{1,2}$ \\ Mei Feng ${ }^{1,2}$ \\ Xuegang Yang ${ }^{3}$ \\ Jie Zhou ${ }^{2}$ \\ $\mathrm{Lu} \mathrm{Li}^{2}$ \\ $\mathrm{Ke} X \mathrm{u}^{2}$ \\ Guohui $\mathrm{Xu}^{1,3, *}$ \\ Jinyi Lang ${ }^{1,2, *}$
}

'School of Medicine, University of Electronic Science and Technology of China, Chengdu, People's Republic of China; ${ }^{2}$ Department of Radiation Oncology, Sichuan Cancer Hospital and Institute, Sichuan Cancer Center, Radiation Oncology Key Laboratory of Sichuan Province, Chengdu, People's Republic of China; ${ }^{3}$ Department of Interventional Radiology, Sichuan Cancer Hospital and Institute, Sichuan Cancer Center, Radiation Oncology Key Laboratory of Sichuan Province, Chengdu, People's Republic of China

*These authors contributed equally to this work

\begin{abstract}
Background: Nasopharyngeal carcinoma (NPC) is one of the most highly radiosensitive malignancies; however, some locally advanced NPC patients experienced local recurrence even though they received aggressive treatment regimens. Defining the tumor volume precisely is important to escalate the total dose required for the primary tumor. In this study, we aimed to investigate the feasibility and efficacy of dose escalation guided by DW-MRI in patients with locally advanced NPC.
\end{abstract}

Patients and Methods: A total of 230 patients with locally advanced NPC treated with intensive modulated radiotherapy (IMRT) at Sichuan Cancer Hospital between January 2010 and January 2015 were enrolled in this retrospective study. All the patients were treated with allcourse of simultaneous integrated boost-IMRT. DW-MRI-guided dose escalation with $2.2-2.5$ Gy/F, qd for 1-3 days or 1.2-1.5 Gy/F, bid for 1-3 days were prescribed to 123 patients. Survival and complication of the patients were evaluated, and multivariate analysis was performed.

Results: The median follow-up of patients in the DW-MRI-guided dose-escalation group and the conventional group was 48 months (range 8-88 months) and 52 months (range 6-90 months), respectively. The 5-year overall survival rate, distant metastasis-free survival rate, progression-free survival, and local recurrence-free survival (LRFS) of patients in the doseescalation group and the conventional group were $88 \%$ vs $82.5 \%(p=0.244), 86.1 \%$ vs $83.3 \%(p=0.741), 82.2 \%$ vs $76.6 \%(p=0.286)$, and $89.1 \%$ vs $80.1 \%(p=0.029)$, respectively. Multivariate analysis showed that dose escalation was independent prognostic factor for LRFS (HR 0.386, 95\% CI 0.163-0.909, $p=0.03$ ).

Conclusion: DW-MRI-guided dose escalation is a feasible strategy to improve local control of patients with locally advanced NPC. The treatment-related complications are tolerable.

Keywords: nasopharyngeal carcinoma, DW-MRI, dose escalation, locally advanced

\section{Background}

Nasopharyngeal carcinoma (NPC) is one of the most highly radiosensitive malignancies, with a growing prevalence year by year in China. ${ }^{1}$ Patients at a locally advanced stage have relatively worse survival than those at an early stage. Although multiple efforts, such as enhancing the intensity of chemotherapy, adding the anti-EGFR drugs, and dose escalation, have been made to improve local control and decrease distant metastasis, ${ }^{2}$ some patients, however, still experienced local recurrence even with such aggressive regimens. Intensive modulated radiotherapy (IMRT) can offer better target coverage and normal organ protection and allow escalation of the radiation dose of gross tumor volume (GTV), ${ }^{3}$ which has been confirmed to improve local control. ${ }^{4}$ Due
Correspondence: Guohui Xu; Jinyi Lang Sichuan Cancer Hospital\& Institute. Sichuan Cancer Center,Radiation Oncology Key Laboratory of Sichuan Province, School of Medicine, University of Electronic Science and Technology of China, No. 55, South Renmin Road, Wuhou District, Chengdu 610000, People's Republic of China

Tel +86-28-85420I52

Emailxgh0913@hotmail.com; langjy610@163.com 
to the radiotherapy complications brought from higher dose, the therapeutic gains from different strategies that escalating the total dose of GTV are unclear and the total dose and time/ fraction are still controversial. ${ }^{2}$

Methods to escalate the total dose of primary tumor usually include altering fraction, sequentially boosting with stereotactic radiotherapy (SRT) or brachytherapy and dose-painting with IMRT. ${ }^{5-7}$ Pan et al found that patients with altered fraction $(1.2 \mathrm{~Gy} / \mathrm{F}$ bid to $48 \mathrm{~Gy}$ followed by 30 Gy in 20 fractions at 1.5 Gy bid) had better local control and overall survival (OS) than the conventional group with $70 \mathrm{~Gy} / 35 \mathrm{~F}$ over 7 weeks in the $2 \mathrm{D}$ radiotherapy era (without chemotherapy, stage was confirmed by CT). ${ }^{5}$ Wang et al showed that patients with bid radiotherapy (RT) regimen had a superiority of 5-year local control, but not in disease-specific and OS, over patients with conventional radiotherapy in T3-4 tumors. ${ }^{8}$ The preliminary results of the NPC-0501 trial showed that accelerated fractionation was not recommended for patients with concurrent chemoradiotherapy (CCRT) in loco-regional advanced stage. ${ }^{9}$ On the other hand, it has been confirmed that dose escalation after the primary course of 66-70 Gy might improve local control (without chemotherapy) of patients in T1/T2a and T3/4 tumors. ${ }^{4}$

The precise definition of the tumor volume, so that it can receive a higher dose, has attracted much attention. Fluorine-18-fluorodeoxyglucose positron emission tomography/computed tomography $\left({ }^{18} \mathrm{~F}\right.$-FDG-PET/CT $)$ or ${ }^{18}$ F-fluoromisonidazole PET-guided dose-escalation has been proven tolerable and valuable for locally advanced NPC $;{ }^{10,11}$ however, the low spatial resolution and accessibility, as well as the affordability of PET-CT in low- and middle-income countries restricted its clinical application. Diffusion-weighted magnetic resonance imaging (DWMRI) and contrast-enhanced MRI, available in most hospitals, which can clearly detect soft tissue circumscription, skull base infiltration, and intracranial extension of NPC, ${ }^{12,13}$ have been widely used as diagnosis, staging, and treatment assessment tool in NPC. In this study, we aimed to compare the effects of DW-MRI-guided dose escalation on survival and local control in NPC patients. This is the first attempt of dose escalation based on DW-MRI for locally advanced NPC.

\section{Patients and Methods}

\section{Patients}

The Institutional Review Board of Sichuan Cancer Hospital approved this retrospective study. Written informed consent was obtained for patients included in the study, which was conducted in accordance with the Declaration of Helsinki. Patients with locally advanced NPC treated with IMRT at Sichuan Cancer Hospital between January 2010 and January 2015 were enrolled in this study. Inclusion criteria for this retrospective study were: 1) 18-65 years old; 2) the pathology was confirmed as WHO type II nasopharyngeal carcinoma; 3) the stage of patients was T3-4N0-3M0; 4) patients had received CCRT with or without neoadjuvant chemotherapy (NAC) with all-course of IMRT; 5) patients had finished the course of radiotherapy with or without DW-MRI-guided dose escalation to primary tumor volume; 6) the ECOG was 0-1, without serious concomitant disease. Conventional written informed consent form was signed for each patient prior to treatment. Patients who received dose escalation needed to approve the regimens and re-signed the written informed consent form for dose escalation. Details of age, gender, $\mathrm{T}$, $\mathrm{N}$, and clinical stage, pathology type, hemoglobin (HGB), radiotherapy dose/fraction, and chemotherapy and targeted therapy regimens were obtained from the medical record system.

\section{Radiotherapy}

All the patients were treated with all-course of simultaneous integrated boost (SIB)-IMRT. The Eclipse ${ }^{\mathrm{TM}}$ Treatment Planning System (Eclipse 11.0, Varian) was used to design and optimize the IMRT radiotherapy planning, which was implemented in the Novalis Tx linear accelerator (NTX, Varian). Thermoplastic masks of head and neck were used to maintain a stable fixation position for CT/MRI simulation and treatment implementation. CT/ MRI fused images were used for target contouring and then CT and RT structure data were transferred to the planning system for planning optimizing. The plan should meet the following conditions: the planning target volume (PTV) should range from $95 \%$ to $110 \%$ of the prescription dose, and the maximum dose of the plan should be in the target volume. The total doses to organs at risk should be limited according to the Radiation Therapy Oncology Group protocol 0225 (RTOG0225).

\section{The Basic SIB-IMRT Course}

All the patients in the study received the basic radiotherapy dose and fraction course as follows: the gross tumor volumes (GTV-nx) D95 2.10-2.25 Gy/F to a total dose of 68.0-72.0 Gy, clinical target volumes (CTV) 1 D95 1.8-2.05 Gy/F to a total dose of 62-64 Gy, CTV2 D95 
$1.7-1.8 \mathrm{~Gy} / \mathrm{F}$ to a total dose of 50-56 Gy; positive neck lymph node (GTV-ln) D95 2.10-2.25 Gy/F to a total dose of 68.0-70.0 Gy; CTVln D95 1.7-1.8 Gy/F to a total dose of 50-56 Gy. The total number of fractions was 28-33.

\section{DW-MRI-Guided Dose Escalation}

All the patients in this study were scheduled with DW-MRI and a contrast-enhanced MRI scan of the nasopharyngeal at the end of the SIB-IMRT course. Patients with a residual lesion on the MRI image had two options, to finish the radiotherapy or to receive dose escalation. An informed consent form regarding which option was made should be resigned, with an explanation of the possible advantages and disadvantages of the two options. The patients' decisions were made after they were told about the possible advantages and disadvantages of dose escalation.

For patients who received DW-MRI-guided dose escalation to the primary tumor, the T2WI, DW-MRI, enhanced
T1WI image and CT image with contrast were merged in the same system and presented on the same screen. The primary tumor volume of dose-escalation was defined on the basis of the multimodal image of MRI and CT. The volume of dose escalation was firstly outlined based on the enhanced region on the TIWI MRI and CT with contrast; afterward, the target was corrected and adjusted based on information on DWI and T2WI (Figure 1). Notably, the necrosis region (hyperintensity on T2WI and hypointensity on T1WI) should be avoided and the diffusion restricted area (hyperintensity on DWI) should be included in the volume of dose-escalation. Patients received dose escalation within one week after finishing the SIB-IMRT course. Prescription dose and fractions for the dose-escalation course were as follows: $2.2-2.5 \mathrm{~Gy} / \mathrm{F}$, qd for 1-3 days for the patients with good response to the former treatment ( $\geq 80 \%$ regression) or $1.2-1.5 \mathrm{~Gy} / \mathrm{F}$, bid for 1-3 days for the patients who are not as sensitive to the former treatment ( $<80 \%$ regression) .
A

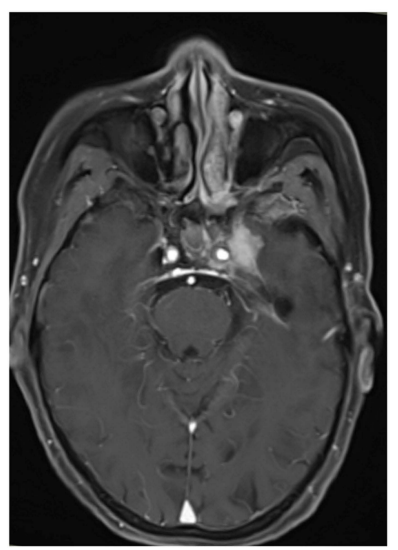

E

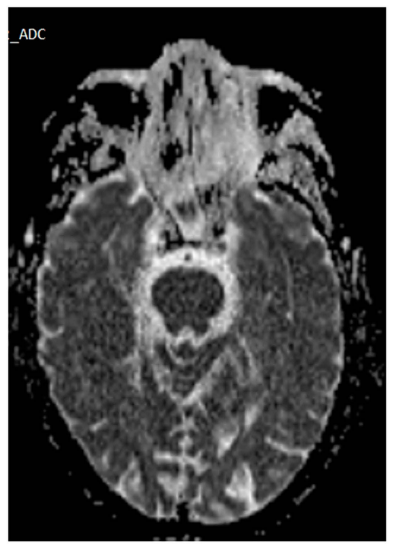

B

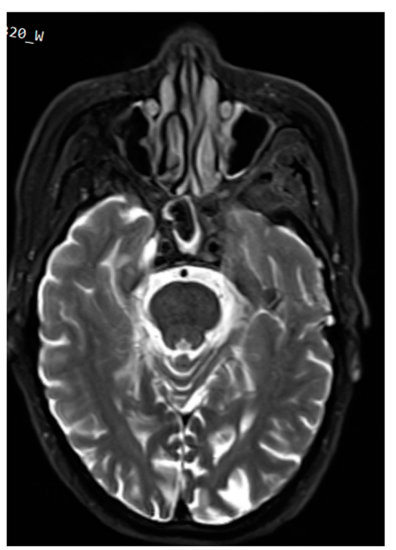

$\mathbf{F}$

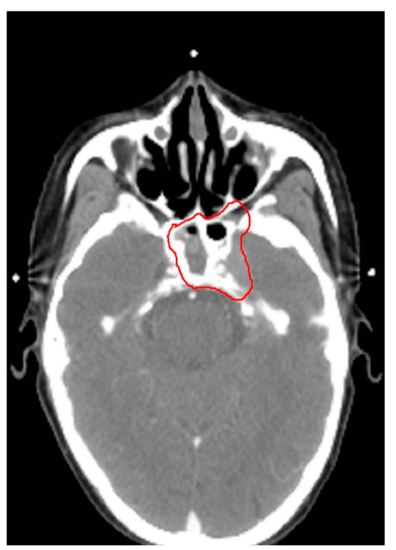

C

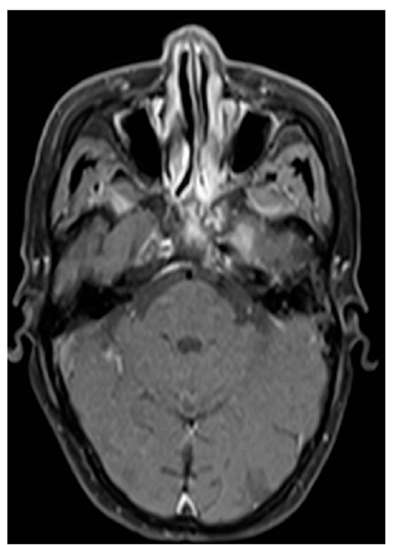

G

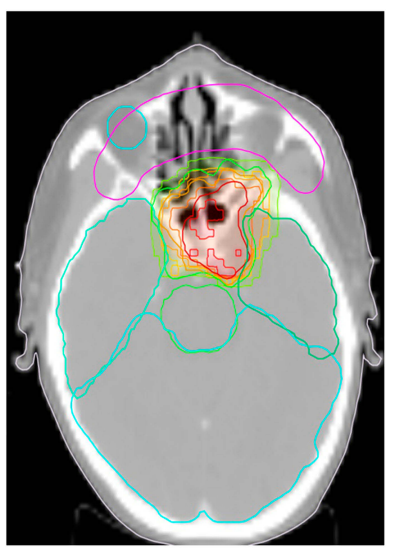

D

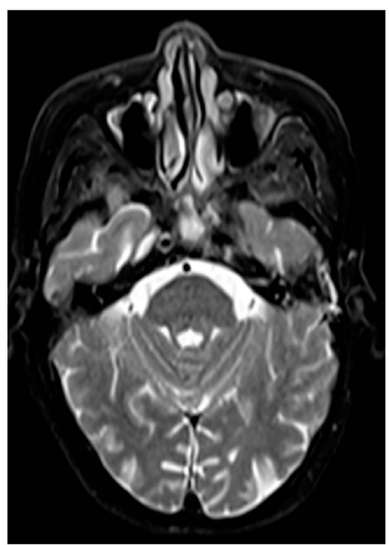

H

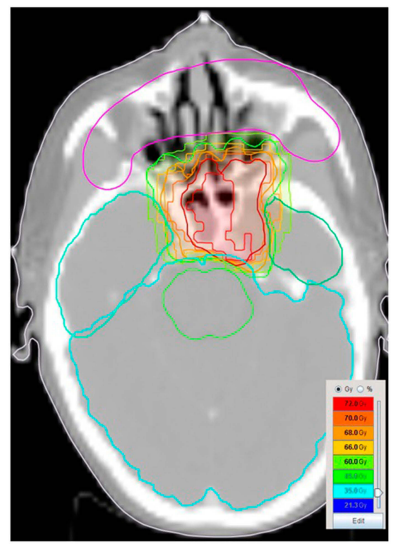

Figure I DW-MRI-guided dose escalation in a 68-year-old NPC patient with T4NIM0. (A) TIWI with contrast prior to radiotherapy. (B) T2WI prior to radiotherapy. (C) TIWI with contrast at $70 \mathrm{~Gy}$. (D) T2WI at $70 \mathrm{~Gy}$. (E) ADC map based on DWI at $70 \mathrm{~Gy}$ (F) CT with contrast at $70 \mathrm{~Gy}$, targets were defined on CT data. (G, H) iso-dose curves on CT imaging, $70 \mathrm{~Gy}$ in red represents areas of $2.2 \mathrm{~Gy}$ per fraction. Dose-escalation prescription for this patient was $2.2 \mathrm{~Gy} / \mathrm{F} * 2 \mathrm{~F}$, the total dose to GTV was 74.8 Gy. 


\section{Chemotherapy and Targeted Therapy}

The regimens of NAC were docetaxel $60 \mathrm{mg} / \mathrm{m}^{2}+$ cisplatin $60 \mathrm{mg} / \mathrm{m}^{2}+$ fluorouracil $600 \mathrm{mg} / \mathrm{m}^{2} \mathrm{~d} 1-5$ (TPF), docetaxel $75 \mathrm{mg} / \mathrm{m}^{2}+$ cisplatin $75-80 \mathrm{mg} / \mathrm{m}^{2}$ (TP) or gemcitabine $1.0 \mathrm{~g} / \mathrm{m}^{2} \mathrm{~d} 1, \mathrm{~d} 8+$ cisplatin $75-80 \mathrm{mg} / \mathrm{m}^{2}$ for 2-3 cycles at intervals of 3 weeks. For CCRT, regimens were cisplatin $80-100 \mathrm{mg} / \mathrm{m}^{2} \mathrm{~d} 1$ or $\mathrm{TP}$, repeated after 21 days for 2-3 cycles.

Anti-EGFR drugs, nimotuzumab or cetuximab, were prescribed in some of the patients. Nimotuzumab was used at a dose of $200 \mathrm{mg}$, every week for 6-8 weeks during radiotherapy. Cetuximab was used at a dose of $400 \mathrm{mg} / \mathrm{m}^{2}$ for the first time and $250 \mathrm{mg} / \mathrm{m}^{2}$ weekly for 6-8 weeks afterward.

\section{Follow-Up}

Follow up was scheduled every 3 months for the first 2 years, every 6 months for the next 3 years, and then annually. The OS was calculated from the date of diagnosis to the date of the death or last follow-up. Distant metastasis-free survival (DMFS) was defined from the day finishing treatment to the day when distant metastasis occurred; progression-free survival (PFS) was calculated from the date for the end of treatment to the date of the local or regional relapse, or distant metastasis. The local relapse-free survival (LRFS) was defined as the time from the end of the treatment to the appearance of local relapse. Physical examination, laboratory examination (including plasma EBV DNA test), nasopharyngoscopy, abdominal B-ultrasound, MRI scan of nasopharynx + neck with contrast, chest X-ray or CT scan and SPECT (annually) should be included in the follow-up. According to the Radiation Therapy Oncology Group (RTOG) criteria, early and late toxicity of radiotherapy were evaluated and recorded.

\section{Statistical Methods}

All statistical analyses were performed using SPSS 22.0 statistical software. The Kaplan Meier method and logrank text were applied to survival analysis. The chi-square test was used to compare the difference between clinical pathological markers and treatment toxicity of each group. Quantitative data were compared with the independent sample $t$ test. The log-rank test was used for univariate analyses. The COX regression model was used for the prognostic analysis of multiple factors. The difference was considered statistically significant when $p<0.05$.

\section{Results}

\section{Characteristics of Patients}

Among the 230 patients included in this study, 127 patients were treated with the base course of chemoradiotherapy plus DW-MRI-guided dose escalation and 103 patients were treated with chemoradiotherapy with the standard dose/fraction. Age, gender, T, N, and clinical stage (all patients were restaged according to American Joint Committee on Cancer (AJCC) 8th edition), pathology type, HGB, chemotherapy regimen (CCRT or NAC+CCRT), cumulative dose of cisplatin and targeted therapy distribution between the DW-MRIguided dose-escalation group and the conventional group were all comparable (Table 1).

Fifty-one out of 57 in the dose-escalation group and 39 out of 46 in the conventional group had skull base invasion. Meanwhile, 67 out of 70 in the dose-escalation group and 52 out of 57 in the conventional group had an intracranial invasion. Of which were comparable between the dose escalation group and conventional group $(p>0.05)$.

Patients in the conventional arm received a total dose of D95 70.9 $\pm 1.3 \mathrm{~Gy}$ in the GTV (T3 70.7 $\pm 1.1 \mathrm{~Gy}$, T4 71.1 $\pm 1.4 \mathrm{~Gy}$, respectively). The total dose of GTV in the DWMRI-guided dose-escalation group was D95 74.3 $\pm 1.4 \mathrm{~Gy}$ (T3, 73.6 $\pm 1.3 \mathrm{~Gy}$; T4 74.8 $\pm 1.2 \mathrm{~Gy}$ ), which was significantly higher than that in the conventional arm $(p<0.05)$. In addition, the doses of GTV in the T3 and T4 subgroup in the dose-escalation arm were significantly higher than that of the conventional arm (Table 2).

The escalation dose was 2.5-6.6 Gy in the qd arm $(n=74)$; among them, 7 patients received $2.5 \mathrm{~Gy} / \mathrm{F}$ for 1 day and 13 patients received $2.2 \mathrm{~Gy} / \mathrm{F}$ for 3 days, while the other patients received 2.2-2.5 Gy/F for 2 days. Meanwhile, the escalation dose was 3-7.2 Gy in the bid arm $(n=53)$, with 5 patients receiving $1.5 \mathrm{~Gy} / \mathrm{F}$ bid for 1 day and 8 patients receiving $1.2 \mathrm{~Gy} / \mathrm{F}$ bid for 3 days; the other patients received $1.2-1.5$ Gy bid for 2 days. All the patients in this study received CCRT. Among them 184 patients received 2-3 cycles of NAC prior to CCRT (99 patients in dose-escalation group and 85 in the conventional group); 33 patients in the doseescalation group (19 patients with nimotuzumab and 14 patients with cetuximab) and 29 patients of the conventional group (16 patients with nimotuzumab and 13 patients with cetuximab) received anti-EGFR treatment.

\section{Survival Outcome}

The median follow-up time in this study was 55 months (range 6-90 months). During the period of follow-up, 14 deaths were 
Table I Clinical-Pathological Characteristics of Patients in the Study

\begin{tabular}{|c|c|c|c|}
\hline Group & $\begin{array}{l}\text { DW-MRI- } \\
\text { Guided Dose } \\
\text { Escalation Arm } \\
\mathrm{N}=1 \mathbf{2 7}(\%)\end{array}$ & $\begin{array}{l}\text { Conventional } \\
\text { Arm N=103 } \\
\text { (\%) }\end{array}$ & $p$ \\
\hline Age (years) & $46.1 \pm 11.6$ & $45.5 \pm 11.0$ & $0.698^{*}$ \\
\hline $\begin{array}{l}\text { Gender } \\
\text { Male } \\
\text { Female }\end{array}$ & $\begin{array}{l}77(60.6) \\
50(39.4)\end{array}$ & $\begin{array}{l}61(59.2) \\
42(40.8)\end{array}$ & $0.829 * *$ \\
\hline $\begin{array}{l}\text { T Stage } \\
\text { T3 } \\
\text { T4 }\end{array}$ & $\begin{array}{l}57(44.9) \\
70(55.1)\end{array}$ & $\begin{array}{l}46(44.7) \\
57(55.3)\end{array}$ & $0.973 * *$ \\
\hline $\begin{array}{c}\text { N Stage } \\
\text { N0-2 } \\
\text { N3 }\end{array}$ & $\begin{array}{l}100(78.7) \\
27(21.3)\end{array}$ & $\begin{array}{l}78(75.7) \\
25(24.3)\end{array}$ & $0.587^{* *}$ \\
\hline $\begin{array}{l}\text { AJCC Stage }^{a} \\
\text { III } \\
\text { IVA }\end{array}$ & $\begin{array}{l}42(33.1) \\
85(66.9)\end{array}$ & $\begin{array}{l}33(32.0) \\
70(68.0)\end{array}$ & $0.868^{* *}$ \\
\hline $\begin{array}{l}\text { Pathology Type } \\
\text { IIA } \\
\text { IIB }\end{array}$ & $\begin{array}{l}47(37.0) \\
80(63.0)\end{array}$ & $\begin{array}{l}45(43.7) \\
58(56.3)\end{array}$ & $0.304 * *$ \\
\hline $\begin{array}{l}\text { HGB } \\
\qquad 140 \mathrm{~g} / \mathrm{L} \\
\geq \mid 40 \mathrm{~g} / \mathrm{L}\end{array}$ & $\begin{array}{l}64(50.4) \\
63(49.6)\end{array}$ & $\begin{array}{l}49(47.6) \\
54(52.4)\end{array}$ & $0.670 * *$ \\
\hline $\begin{array}{l}\text { EBV-DNA } \\
\qquad 400 \mathrm{copy} / \mathrm{mL} \\
<400 \mathrm{copy} / \mathrm{mL}\end{array}$ & $\begin{array}{l}19(15.0) \\
108(85.0)\end{array}$ & $\begin{array}{l}13(12.6) \\
90(87.4)\end{array}$ & $\begin{array}{l}0.260 * * \\
0.389 * *\end{array}$ \\
\hline $\begin{array}{l}\text { Treatment option } \\
\text { CCRT } \\
\text { NAC+CCRT }\end{array}$ & $\begin{array}{l}28(22.1) \\
99(77.9)\end{array}$ & $\begin{array}{l}18(17.5) \\
85(82.5\end{array}$ & \\
\hline $\begin{array}{l}\text { Cumulative CDDP } \\
<200 \mathrm{mg} / \mathrm{m}^{2} \\
\geq 200 \mathrm{mg} / \mathrm{m}^{2}\end{array}$ & $\begin{array}{l}56(44.1) \\
7 I(55.9)\end{array}$ & $\begin{array}{l}4 I(39.8) \\
62(60.2)\end{array}$ & $0.513^{* *}$ \\
\hline
\end{tabular}

Notes: ${ }^{*} p$-values were calculated with an independent sample $t$-test; ${ }^{*} p$-values

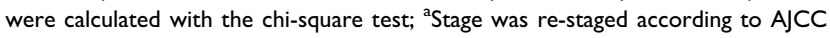
8th edition based on MRI.

observed in the dose-escalation group and 17 deaths were observed in the conventional group. Eight patients experienced local recurrence in the dose-escalation group while 13 patients experienced local recurrence in the conventional group. The median time-to-local recurrence was 43 months (range 26-56 months) in the dose-escalation group and 36 months (range 19-52 months) the conventional group, respectively. Seventeen patients developed distant metastasis in the dose-escalation group and 13 patients developed distant metastasis in the conventional group.
Table 2 Cumulative Dose of Gross Tumor Volume (GTV) in Each Group

\begin{tabular}{|c|l|l|l|}
\hline Group & $\begin{array}{l}\text { DW-MRI- } \\
\text { Guided } \\
\text { Dose } \\
\text { Escalation } \\
\text { Arm (Gy) }\end{array}$ & $\begin{array}{l}\text { Conventional } \\
\text { Arm (Gy) }\end{array}$ & $p^{*}$ \\
\hline The whole group & $74.3 \pm 1.4$ & $70.9 \pm 1.3$ & $<0.001$ \\
\hline $\begin{array}{c}\text { Subgroup by T Stage } \\
\text { T3 }\end{array}$ & $\begin{array}{l}73.6 \pm 1.3 \\
\text { T4 }\end{array}$ & $\begin{array}{l}70.7 \pm 1.1 \\
71.1 \pm 1.4\end{array}$ & $\begin{array}{l}<0.001 \\
<0.001\end{array}$ \\
\hline
\end{tabular}

Note: ${ }^{*} p$-values were calculated with an independent sample $t$-test.

The 5-year OS, DMFS, PFS, and LRFS of patients in the dose-escalation group and the conventional group were $88 \%$ vs $82.5 \%$ ( $p=0.244), 86.1 \%$ vs $83.3 \%(p=0.741), 82.2 \%$ vs $76.6 \%(p=0.286)$ and $89.1 \%$ vs $80.1 \%(p=0.029)$, respectively (Figure 2). Subgroup survival analysis of T3 patients showed that the 5-year OS, DMFS, PFS, and LRFS of the dose-escalation group and the conventional group were $94.7 \%$ vs $90.6 \% \quad(p=0.223), 94.1 \%$ vs $84.9 \% \quad(p=0.483)$, $89.4 \%$ vs $79.7 \%(p=0.468)$ and $94.7 \%$ vs $90.6 \%(p=0.223)$, respectively. Meanwhile, the 5-year OS, DMFS, PFS, and LRFS of patients with T4 in the dose-escalation group and the conventional group were $83.7 \%$ vs $75.4 \% \quad(p=0.473)$, $77.1 \%$ vs $87.4 \%(p=0.333), 76.8 \%$ vs $75.1 \%(p=0.444)$ and $85.5 \%$ vs $79.5 \%(p=0.037)$, respectively (Table 3$)$.

\section{Univariate and Multivariate Analysis}

Univariate and multivariate analysis showed that dose escalation was independent prognostic factor for LRFS (HR 0.386, 95\% CI 0.163-0.909, $p=0.03$ ). Clinical stage was an independent prognostic factor for LRFS (HR 3.862, 95\% CI 1.146-13.02, $p=0.029$ ), PFS (HR 2.3386, 95\% CI 1.050-5.421, $p=0.038$ ), DMFS (HR 2.758, 95\% CI 1.059-7.184, $p=0.038)$ and OS (HR 3.509, 95\% CI 1.288-10.030, $p=0.019), \mathrm{N}$ stage was an independent prognostic factor for DMFS (HR 2.302, 95\% CI 1.2035.452, $p=0.043$ ) (Table 4).

\section{RT-Related Complications}

The main early radiation-related complications included mucositis, dysphagia, skin reaction, and xerostomia. The incidence rates of grade III/IV mucositis in the doseescalation group and the conventional group were $54.3 \%$ and $43.7 \%$, respectively. Additionally, $22.0 \%, 14.2 \%$, and $8.7 \%$ of patients in the dose-escalation group experienced 

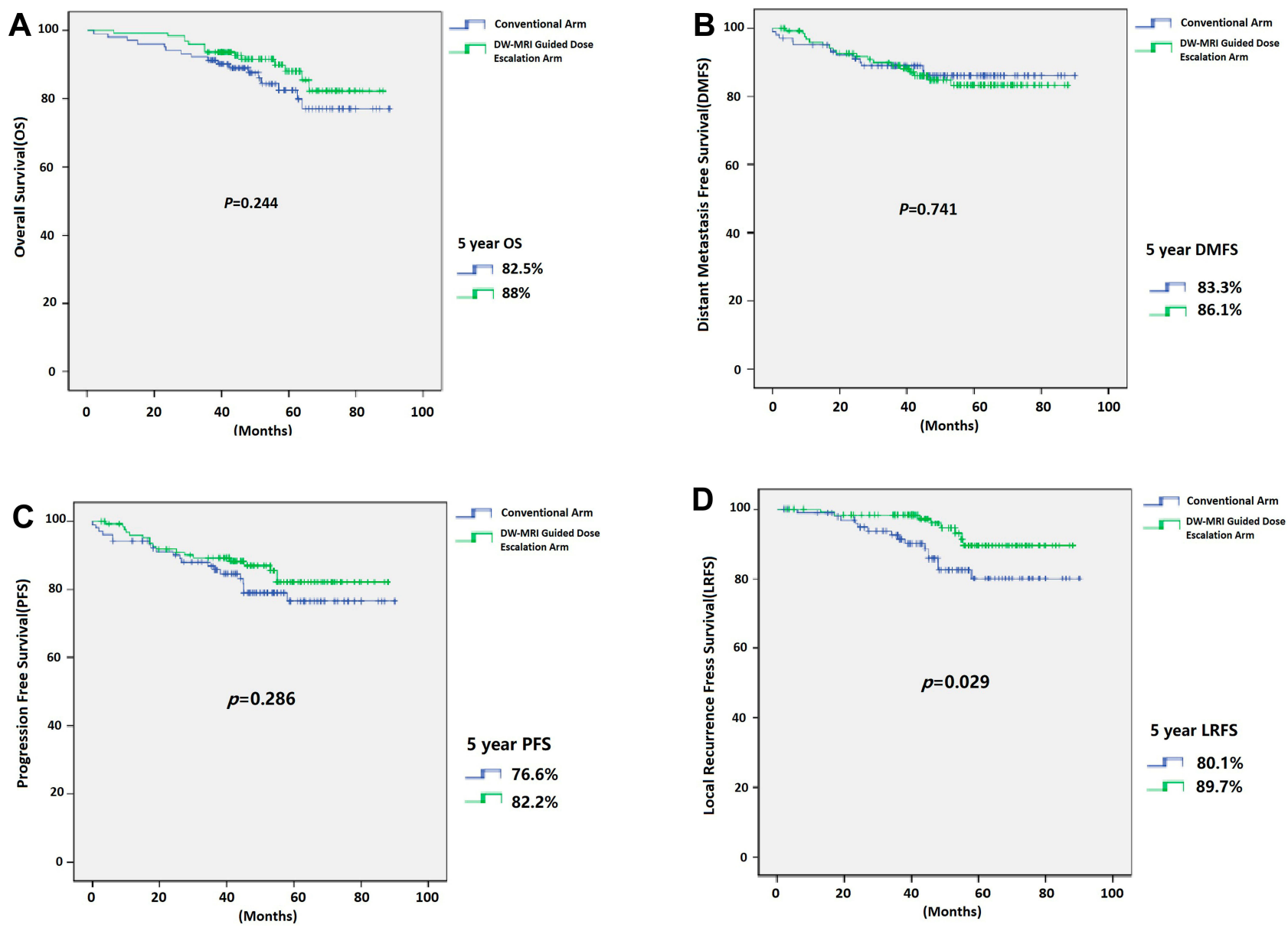

Figure 2 Survival curve of patients in the conventional arm and dose-escalation arm. Abbreviations: OS, overall survival; DMFS, distant metastasis-free survival; PFS, progression-free survival; LRFS, local relapse-free survival.

grade 3 dysphagia, skin reaction, and xerostomia; $15.5 \%$, $10.7 \%$, and $3.9 \%$ of patients in the conventional group experienced grade 3 dysphagia, skin reaction, and xerostomia.

No grade III or higher late complications were observed in the study. Of patients in the dose-escalation group and conventional group, $27.6 \%$ and $33 \%$, respectively, developed late grade II xerostomia. Of patients in the dose-escalation group and the conventional group, $5.5 \%$ and $3.9 \%$, respectively, experienced temporal lobe necrosis (TLN) (grade I). Of patients in the doseescalation group, $19.7 \%, 37.8 \%$, and $7.1 \%$ developed grade I skin fibrosis, hearing loss, and hypopsia, respectively, while $33.0 \%, 14.6$, and $7.8 \%$ of patients in the conventional group developed grade I skin fibrosis, hearing loss and hypopsia, respectively. Overall, the incidence rates of early or late complications between the two groups were not significantly different $(p>0.05)$ (Table 5).

\section{Discussion}

Research on dose escalation of NPC has a relatively long history. Early in 1997, Cmelak et al found that a stereotactic radiosurgical boost of 12 Gy (range 7-16 Gy) following a fractionated dose of 66 Gy (range 64.8-70 Gy) without chemotherapy for locally advanced NPC provided excellent local control. ${ }^{14} \mathrm{Zi}$-qiang Pan et al confirmed that late course accelerated hyper-fractionated radiotherapy achieved higher local control rates and OS than conventionally fractionated radiotherapy. ${ }^{5}$ However, most of the studies regarding dose escalation were based on two-dimensional radiotherapy (2D-RT) or on patients without concurrent chemotherapy. ${ }^{4,5,14}$ Notably, in the IMRT era, the survival of locally advanced NPC was significantly longer than that in 2D-RT or 3D-conformal RT era, ${ }^{3,15,16}$ and CCRT was considered as the standard optimal regimen. ${ }^{17}$ However, strategies of dose escalation for locally advanced NPC with CCRT in the IMRT era are 
Table 3 Subgroup Survival Analysis of T3 and T4

\begin{tabular}{|c|l|l|l|}
\hline Survival & $\begin{array}{l}\text { DW-MRI-Guided Dose } \\
\text { Escalation Arm }\end{array}$ & $\begin{array}{l}\text { Conventional } \\
\text { Arm }\end{array}$ & $\boldsymbol{p}^{*}$ \\
\hline OS & & & \\
T3 & $94.7 \%$ & $90.6 \%$ & 0.223 \\
T4 & $83.7 \%$ & $75.4 \%$ & 0.473 \\
\hline DMFS & & & \\
T3 & $94.1 \%$ & $84.9 \%$ & 0.483 \\
T4 & $77.1 \%$ & $87.4 \%$ & 0.333 \\
\hline PFS & & & \\
T3 & $89.4 \%$ & $79.7 \%$ & 0.468 \\
T4 & $76.8 \%$ & $75.1 \%$ & 0.444 \\
\hline LRFS & & & \\
T3 & $94.7 \%$ & $90.6 \%$ & 0.223 \\
T4 & $85.5 \%$ & $79.5 \%$ & $0.037 * *$ \\
\hline
\end{tabular}

Notes: ${ }^{p}$-values were calculated with the log rank test. $*$ LRFS in T4 patients who received dose escalation was significantly higher than that of patients who received conventional dose.

not well understood but may provide important clues to further improve tumor control and OS of these patients.

In this study, we aim to further explore the feasibility and value of CCRT plus DWI-MRI-guided dose escalation for locally advanced NPC with IMRT. We found that the local recurrence-free survival of the dose-escalation group (especially in patients with T4 stage) was significantly higher than that of the SIM-IMRT group without boost ( $89.1 \%$ vs $80.1 \%, p=0.029)$. Multivariate analysis showed that the clinical stage and the dose escalation were independent prognostic factors for LRFS. The HR of dose escalation is $0.386(95 \%$ CI $0.163-0.909, p=0.03)$, indicating that dose escalation is a protection factor for reducing local recurrence.

The most widely accepted dose fraction regimens of NPC are sequential IMRT with a single fraction of 2.0 Gy over 35 fractions or SIB-IMRT with a single fraction of 2.12-2.36 Gy over $27-35$ fractions. ${ }^{2,18}$ For patients with bigger GTV ( $\geq 64 \mathrm{~mL}$ ) or with the invasion of the skull base or brain, it is preferred to give a higher radiation dose of more than $72 \mathrm{~Gy} .{ }^{19}$ Dose/fraction of escalation in the history ranged from 6 to 15 Gy in a single fraction or 8 to 15 Gy in 3-5 fractions. ${ }^{2}$ Kwong et al found that he GTV received 2.17 Gy daily to a total dose of 76 Gy with simultaneous modulated accelerated-radiation therapy boost technique is feasible with T3-T4 NPC and can be combined with chemotherapy. ${ }^{20}$ However, the study only explored the feasibility of the dose/technique with a 2-year follow-up; there were no further reports on survival and complications for those patients. In this study, all the patients received CCRT, most of the patients (184) received NAC prior to CCRT, a small number of patients (62) even received anti-EGFR targeted treatment, which was a relatively strong treatment regimen. Given that a higher dose of escalation might result in more serious complications that offset the local control benefit from dose-escalation, we prescribed a boost dose of 2.2-2.5 $\mathrm{Gy} / \mathrm{F}$, qd for $1-3$ days or $1.2-1.5 \mathrm{~Gy} / \mathrm{F}$, bid for 1-3 days; the total dose of our study $(74.3 \pm 1.4 \mathrm{~Gy})$ was in line with the dose reported by Kam et al in 2004. Patients (T2b-T4) in the study received $8 \mathrm{~Gy} / 4$ fractions boost after the first course with 66 Gy to the GTV (total dose, $74 \mathrm{~Gy}$ ). ${ }^{21}$

Dose escalation beyond 70 Gy may increase the incidence of RT complications. The target volume of dose escalation is required to be accurate and narrow to ensure the safety of the treatment. The application of MRI, together with the positioning of $\mathrm{CT}$ in the same gesture with thermoplastic masks, improved the accuracy and safety of radiotherapy in head and neck cancer. Adding DWI to the contrast MRI can better differentiate active tumor tissues from necrosis or inflammatory regions and help to delineate target localization. ${ }^{13,22}$ In this study, we found the incidence rate of TLN in the dose-escalation group was $5.5 \%$, which was slightly higher but not significantly than the group without boost (3.9\%). With strict restriction to the normal temporal lobe, the incidence rate of TLN was lower than that of the data we have published before. ${ }^{23}$ In this regard, DW-MRI is a feasible, accessible, and affordable imaging method to guide the precise definition of GTV delineation, which is worthy of clinical application and further study.

Patients' OS in the dose-escalation group was not statistically higher than in the conventional group $(88 \%$ vs $82.5 \%, p=0.244)$. Multivariate analysis showed that the clinical stage (restaged according to AJCC 8th edition), not the dose escalation, was the independent prognostic factor for OS. However, the survival curve of patients in the dose-escalation group is separated from that of patients in the conventional group from the third year after radiotherapy (Figure 2). Distant metastasis was still the predominant pattern of failure. The benefit of dose escalation on OS might be overlapped by distant metastasis or other patterns of failure. Further prospective, large sample, randomized control studies on dose escalation of locally advanced NPC may highlight the benefit of dose escalation on OS in the future. 
Table 4 Univariate and Multivariate Analyses of Prognostic Factors for Different Outcomes

\begin{tabular}{|c|c|c|c|c|c|c|c|c|c|c|}
\hline & \multicolumn{5}{|l|}{ LRFS } & \multicolumn{5}{|c|}{ DMFS } \\
\hline & \multicolumn{2}{|c|}{ Univariate Analysis } & \multicolumn{3}{|c|}{ Multivariate Analysis } & \multicolumn{2}{|c|}{ Univariate Analysis } & \multicolumn{3}{|c|}{ Multivariate Analysis } \\
\hline & $x^{2}$ & $p^{*}$ & HR & $95 \% \mathrm{Cl}$ & $p^{* *}$ & $x^{2}$ & $\mathbf{p}^{*}$ & HR & $95 \% \mathrm{Cl}$ & $p^{* *}$ \\
\hline Gender & 0.169 & 0.681 & & & & 0.008 & 0.930 & & & \\
\hline Age & 2.038 & 0.153 & & & & 0.79 & 0.778 & & & \\
\hline Pathology type & 0.174 & 0.677 & & & & 0.612 & 0.434 & & & \\
\hline HGB & 0.182 & 0.670 & & & & 1.095 & 0.295 & & & \\
\hline T stage & 1.353 & 0.245 & & & & 2.344 & 0.126 & & & \\
\hline$N$ stage & 0.651 & 0.420 & & & & 4.397 & 0.036 & 2.302 & $1.203-5.452$ & 0.043 \\
\hline Clinical stage & 5.211 & 0.022 & 3.862 & $1.146-13.02$ & 0.029 & 4.916 & 0.027 & 2.758 & $1.059-7.184$ & 0.038 \\
\hline EBV-DNA & 2.411 & 0.120 & & & & 2.602 & 0.107 & & & \\
\hline Treatment option & 0.163 & 0.686 & & & & 0.201 & 0.654 & & & \\
\hline Cumulative CDDP & 0.360 & 0.549 & & & & 0.937 & 0.333 & & & \\
\hline \multirow[t]{4}{*}{ Dose escalation } & 10.276 & 0.006 & 0.385 & $0.163-0.909$ & 0.030 & 1.168 & 0.280 & & & \\
\hline & \multicolumn{5}{|l|}{ PFS } & \multicolumn{5}{|l|}{ os } \\
\hline & \multicolumn{2}{|c|}{ Univariate Analysis } & \multicolumn{3}{|c|}{ Multivariate Analysis } & \multicolumn{2}{|c|}{ Univariate Analysis } & \multicolumn{3}{|c|}{ Multivariate Analysis } \\
\hline & $x^{2}$ & $p^{*}$ & HR & $95 \% \mathrm{Cl}$ & $p^{* *}$ & $x^{2}$ & $\mathbf{p}^{*}$ & HR & $95 \% \mathrm{Cl}$ & $p^{* *}$ \\
\hline Gender & 0.017 & 0.896 & & & & 0.030 & 0.863 & & & \\
\hline Age & 0.355 & 0.552 & & & & 0.600 & 0.439 & & & \\
\hline Pathology type & 0.695 & 0.404 & & & & 0.301 & 0.583 & & & \\
\hline HGB & 0.965 & 0.326 & & & & 1.469 & 0.225 & & & \\
\hline T stage & 0.120 & 0.729 & & & & 0.047 & 0.829 & & & \\
\hline $\mathrm{N}$ stage & 2.218 & 0.145 & & & & 1.825 & 0.177 & & & \\
\hline Clinical stage & 4.985 & 0.026 & 2.386 & $1.050-5.421$ & 0.038 & 6.247 & 0.012 & 3.509 & $1.288-10.030$ & 0.019 \\
\hline EBV-DNA & 2.843 & 0.092 & & & & 0.523 & 0.470 & & & \\
\hline Treatment option & 0.315 & 0.574 & & & & 0.935 & 0.334 & & & \\
\hline Cumulative CDDP & 1.619 & 0.203 & & & & 0.284 & 0.594 & & & \\
\hline Dose escalation & 1.517 & 0.218 & & & & 1.596 & 0.207 & & & \\
\hline
\end{tabular}

Notes: * $p$-values were calculated with the log rank test. ** $p$-values were calculated with a forward Cox proportional-hazards model.

Abbreviations: $\mathrm{HR}$, hazard ratio; $95 \% \mathrm{Cl}, 95 \%$ confidence interval.

Table 5 Early and Late Treatment Toxicity

\begin{tabular}{|c|c|c|c|c|c|c|c|c|c|c|c|c|}
\hline \multirow[t]{2}{*}{ Grade } & \multicolumn{5}{|c|}{$\begin{array}{l}\text { DW-MRI-Guided Dose Escalation Arm } \\
(\mathrm{N}=\text { I 27) } N(\%)\end{array}$} & \multicolumn{5}{|c|}{ Conventional Arm (N=103) N (\%) } & \multirow[t]{2}{*}{$\begin{array}{l}\chi^{2} \\
\text { value }\end{array}$} & \multirow[t]{2}{*}{$\mathbf{p}^{*}$} \\
\hline & 0 & $\mathbf{I}$ & II & III & IV & 0 & $\mathbf{I}$ & II & III & IV & & \\
\hline \multicolumn{13}{|l|}{ Early } \\
\hline Mucositis & $0(0)$ & 10(7.9) & $48(37.8)$ & $63(49.6)$ & $6(4.7)$ & $0(0)$ & $13(12.6)$ & $45(43.7)$ & $4 I(39.8)$ & $4(3.9)$ & 3.071 & 0.381 \\
\hline Dysphagia & $\mid 4(|| .0)$ & $18(14.1)$ & $67(52.8)$ & $28(22.0)$ & $0(0)$ & $\mathrm{II}(10.7)$ & $15(14.6)$ & $6 I(59.2)$ & $16(15.5)$ & $0(0)$ & $\mathrm{I} .70 \mathrm{I}$ & 0.637 \\
\hline Skin reaction & $0(0)$ & $33(26.0)$ & $76(59.8)$ & $18(14.2)$ & $0(0)$ & $0(0)$ & $37(35.9)$ & $55(53.4)$ & $\mathrm{II}(10.7)$ & $0(0)$ & 2.811 & 0.245 \\
\hline Xerostomia & $0(0)$ & $39(30.7)$ & $77(60.6)$ & II(8.7) & $0(0)$ & $0(0)$ & $39(37.9)$ & $60(58.2)$ & $4(3.9)$ & $0(0)$ & 2.903 & 0.234 \\
\hline \multicolumn{13}{|l|}{ Late } \\
\hline Xerostomia & $39(30.7)$ & $53(4 I .7)$ & $35(27.6)$ & $0(0)$ & $0(0)$ & $42(40.8)$ & $34(33.0)$ & $27(26.2)$ & $0(0)$ & $0(0)$ & 2.819 & 0.244 \\
\hline Skin fibrosis & $102(80.3)$ & $25(19.7)$ & $0(0)$ & $0(0)$ & $0(0)$ & $88(85.4)$ & $15(14.6)$ & $0(0)$ & $0(0)$ & $0(0)$ & 1.039 & 0.308 \\
\hline Hearing loss & $79(62.2)$ & $48(37.8)$ & $0(0)$ & $0(0)$ & $0(0)$ & $60(58.3)$ & $43(4 I .7)$ & $0(0)$ & $0(0)$ & $0(0)$ & 0.372 & 0.542 \\
\hline Brain necrosis & $120(94.5)$ & $7(5.5)$ & $0(0)$ & $0(0)$ & $0(0)$ & $99(96.1)$ & $4(3.9)$ & $0(0)$ & $0(0)$ & $0(0)$ & 0.243 & 0.622 \\
\hline Hypopsia & $118(92.9)$ & $9(7.1)$ & $0(0)$ & $0(0)$ & $0(0)$ & $95(92.2)$ & $8(7.8)$ & $0(0)$ & $0(0)$ & $0(0)$ & 0.006 & 0.940 \\
\hline
\end{tabular}

Note: ${ }^{*} p$-values were calculated by the chi-square test. 
There are still some intrinsic limitations of this retrospective study. Firstly, the selection bias was not avoided. In addition, the inconsistency of the dose/fraction of boost and chemotherapy as well as targeted therapy regimens might confuse the benefit of dose escalation. Secondly, long-term follow-up is needed to observe late complications. Despite these limitations, this study still provides useful suggestions for the treatment of locally advanced NPC in the era of IMRT. To our knowledge, this is the first study to evaluate the feasibility and value of dose escalation guided by DWI-MRI for locally advanced NPC.

\section{Conclusion}

This study confirmed that DW-MRI-guided dose escalation is a feasible strategy to improve local control of locally advanced NPC patients in the era of IMRT. The radiation-related complications are tolerable. Further large-sample, prospective, randomized clinical studies are warranted.

\section{Abbreviations}

NPC, nasopharyngeal carcinoma; IMRT, intensitymodulated radiotherapy; CCRT, concurrent chemoradiotherapy; NAC, neoadjuvant chemotherapy; GTV, gross tumor volume; OS, overall survival; DMFS, distant metastasis-free survival; PFS, progression-free survival; LRFS, local relapse free-survival; DW-MRI, diffusion weighted magnetic resonance imaging; FDG-PET/CT, positron emission tomography/computed tomography; SRT, stereotactic radiotherapy.

\section{Data Sharing Statement}

Please contact the corresponding author for data requests.

\section{Ethics and Consent Statements}

The Institutional Review Board of Sichuan Cancer Hospital approved this retrospective study. This study was conducted in accordance with the Declaration of Helsinki.

\section{Consent for Publication}

Written informed consent was obtained before treatment for patients included in the study.

\section{Author Contributions}

All authors contributed to data analysis, drafting or revising the article, gave final approval of the version to be published, and agree to be accountable for all aspects of the work.

\section{Funding}

The study was supported by the National Key Research and Development Plan no. 2017YFC0113100 and the Sichuan Science and Technology Plan Project, no. 18YYJC0665.

\section{Disclosure}

The authors state that there are no conflicts of interest.

\section{References}

1. Wei KR, Zheng RS, Zhang SW, Liang ZH, Li ZM, Chen WQ. Nasopharyngeal carcinoma incidence and mortality in China, 2013. Chin J Cancer. 2017;36(1):90. doi:10.1186/s40880-017-0257-9

2. Kam MK, Wong FC, Kwong DL, Sze HC, Lee AW. Current controversies in radiotherapy for nasopharyngeal carcinoma (NPC). Oral Oncol. 2014;50(10):907-912. doi:10.1016/j.oraloncology.2013.09.013

3. Kam MK, Chau RM, Suen J, Choi PH, Teo PM. Intensity-modulated radiotherapy in nasopharyngeal carcinoma: dosimetric advantage over conventional plans and feasibility of dose escalation. Int J Radiat Oncol Biol Phys. 2003;56(1):145-157. doi:10.1016/ S0360-3016(03)00075-0

4. Teo PM, Leung SF, Tung SY, et al. Dose-response relationship of nasopharyngeal carcinoma above conventional tumoricidal level: a study by the Hong Kong nasopharyngeal carcinoma study group (HKNPCSG). Radiother Oncol. 2006;79(1):27-33. doi:10.1016/j. radonc.2006.03.012

5. Pan ZQ, He XY, Guo XM, et al. A Phase III study of late course accelerated hyperfractionated radiotherapy versus conventionally fractionated radiotherapy in patients with nasopharyngeal carcinoma. Am J Clin Oncol. 2012;35(6):600-605. doi:10.1097/ COC.0b013e31822dfd55

6. Shan GP, Wang BB, Zheng P, Du FL, Yang YW. Efficacy and safety of chemotherapy combined with stereotactic radiotherapy in the treatment of nasopharyngeal carcinoma. Med Sci Monit. 2017;23:5630-5636. doi:10.12659/MSM.903903

7. Levendag PC, Keskin-Cambay F, de Pan C, et al. Local control in advanced cancer of the nasopharynx: is a boost dose by endocavitary brachytherapy of prognostic significance? Brachytherapy. 2013;12 (1):84-89. doi:10.1016/j.brachy.2012.06.001

8. Lee AW, Sze WM, Yau TK, Yeung RM, Chappell R, Fowler JF. Retrospective analysis on treating nasopharyngeal carcinoma with accelerated fractionation (6 fractions per week) in comparison with conventional fractionation (5 fractions per week): report on 3-year tumor control and normal tissue toxicity. Radiother Oncol. 2001;58 (2):121-130. doi:10.1016/S0167-8140(00)00312-1

9. Lee AW, Ngan RK, Tung SY, et al. Preliminary results of trial NPC-0501 evaluating the therapeutic gain by changing from concurrent-adjuvant to induction-concurrent chemoradiotherapy, changing from fluorouracil to capecitabine, and changing from conventional to accelerated radiotherapy fractionation in patients with locoregionally advanced nasopharyngeal carcinoma. Cancer. 2015;121(8):1328-1338. doi:10.1002/cncr.29208

10. Wang J, Zheng J, Tang T, et al. A randomized pilot trial comparing Position Emission Tomography (PET)-guided dose escalation radiotherapy to conventional radiotherapy in chemoradiotherapy treatment of locally advanced nasopharyngeal carcinoma. PLoS One. 2015;10 (4):e0124018. doi:10.1371/journal.pone.0124018

11. Qiu J, Lv B, Fu M, Wang X, Zheng X, Zhuo W. (18) F-Fluoromisonidazole positron emission tomography/CT-guided volumetric-modulated arc therapy-based dose escalation for hypoxic subvolume in nasopharyngeal carcinomas: a feasibility study. Head Neck. 2017;39(12):2519-2527. doi:10.1002/hed.24925 
12. Chang JT, Lin CY, Chen TM, et al. Nasopharyngeal carcinoma with cranial nerve palsy: the importance of MRI for radiotherapy. Int J Radiat Oncol Biol Phys. 2005;63(5):1354-1360. doi:10.1016/j. ijrobp.2005.05.042

13. Chen Y, Liu X, Zheng D, et al. Diffusion-weighted magnetic resonance imaging for early response assessment of chemoradiotherapy in patients with nasopharyngeal carcinoma. Magn Reson Imaging. 2014;32(6):630-637. doi:10.1016/j.mri.2014.02.009

14. Cmelak AJ, Cox RS, Adler JR, Fee WE Jr., Goffinet DR. Radiosurgery for skull base malignancies and nasopharyngeal carcinoma. Int J Radiat Oncol Biol Phys. 1997;37(5):997-1003. doi:10.1016/S0360-3016(97)00111-9

15. Chen L, ZhangY, Lai SZ, et al. 10-year results of therapeutic ratio by intensity-modulated radiotherapy versus two-dimensional radiotherapy in patients with nasopharyngeal carcinoma. Oncologist. 2018;24(1).

16. Lee AW, Ng WT, Chan LL, et al. Evolution of treatment for nasopharyngeal cancer-success and setback in the intensity-modulated radiotherapy era. Radiother Oncol. 2014;110(3):377-384. doi:10.10 16/j.radonc.2014.02.003

17. Yu Y, Liang H, Lv X, et al. Platinum-based concurrent chemotherapy remains the optimal regimen for nasopharyngeal carcinoma: a large institutional-based cohort study from an endemic area. J Cancer Res Clin Oncol. 2018. doi:10.1007/s00432-018-2721-6
18. Xiao WW, Huang SM, Han F, et al. Local control, survival, and late toxicities of locally advanced nasopharyngeal carcinoma treated by simultaneous modulated accelerated radiotherapy combined with cisplatin concurrent chemotherapy: long-term results of a Phase 2 study. Cancer. 2011;117(9):1874-1883. doi:10.1002/cncr.25754

19. Willner J, Baier K, Pfreundner L, Flentje M. Tumor volume and local control in primary radiotherapy of nasopharyngeal carcinoma. Acta Oncol. 1999;38(8):1025-1030. doi:10.1080/028418699432301

20. Kwong DL, Sham JS, Leung LH, et al. Preliminary results of radiation dose escalation for locally advanced nasopharyngeal carcinoma. Int J Radiat Oncol Biol Phys. 2006;64(2):374-381. doi:10.1016/j. ijrobp.2005.07.968

21. Kam MK, Teo PM, Chau RM, et al. Treatment of nasopharyngeal carcinoma with intensity-modulated radiotherapy: the Hong Kong experience. Int J Radiat Oncol Biol Phys. 2004;60(5):1440-1450. doi:10.1016/j.ijrobp.2004.05.022

22. Ginat DT, Mangla R, Yeaney G, Johnson M, Ekholm S. Diffusionweighted imaging for differentiating benign from malignant skull lesions and correlation with cell density. AJR Am J Roentgenol. 2012;198(6):W597-W601. doi:10.2214/AJR.11.7424

23. Feng M, Huang Y, Fan X, Xu P, Lang J, Wang D. Prognostic variables for temporal lobe injury after intensity modulated-radiotherapy of nasopharyngeal carcinoma. Cancer Med. 2018;7(3):557-564. doi:10.1002/ cam4.1291

\section{Publish your work in this journal}

Cancer Management and Research is an international, peer-reviewed open access journal focusing on cancer research and the optimal use of preventative and integrated treatment interventions to achieve improved outcomes, enhanced survival and quality of life for the cancer patient.
The manuscript management system is completely online and includes a very quick and fair peer-review system, which is all easy to use. Visit http://www.dovepress.com/testimonials.php to read real quotes from published authors. 